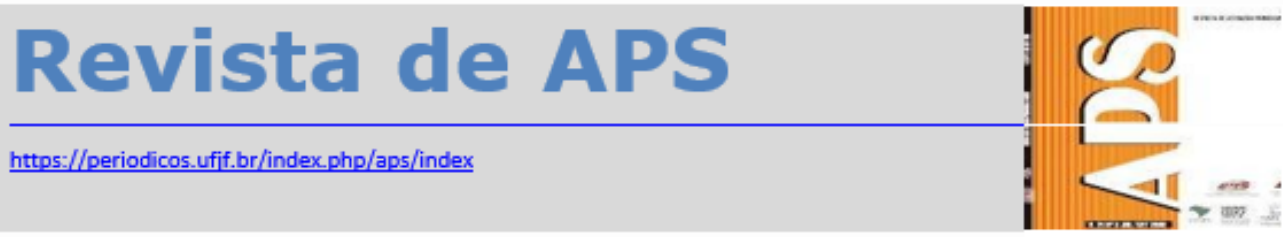

\title{
Formação, provimento e fixação na Estratégia Saúde da Família de um município do Nordeste brasileiro
}

\author{
Formation, provision and placement of the Family Health Strategy of a \\ municipality in the Northeast Brazil
}

\author{
Bruno Oliveira de Aguiar Santos, ${ }^{1}$ Ana Paula Soares Gondim, ${ }^{2}$ Ana Carolina Santana \\ Praxedes ${ }^{3}$
}

RESUMO

A formação, o provimento e a fixação dos médicos na Estratégia Saúde da Família (ESF) são pontos relevantes para o fortalecimento da Atenção Básica no Brasil. 0 objetivo do trabalho é avaliar a formação e os fatores relacionados ao provimento e à fixação dos médicos da ESF de um município do Nordeste do Brasil, estabelecendo um comparativo com dentistas e enfermeiros. Trata-se de estudo descritivo quantitativo com aplicação de questionário estruturado com 119 profissionais de saúde. Os médicos apresentaram bom índice (68\%) de especialização em saúde da família, tempo médio na ESF de 5,2 anos, além de considerarem a bonificação para prova de títulos e a necessidade financeira as principais razões para atuação na ESF. Oitenta por cento da categoria médica advinha do Programa Mais Médicos, sendo esta a grande diferença entre os profissionais não médicos, que são concursados em sua maioria.

PALAVRAS-CHAVE: Atenção Básica. Formação profissional em saúde. Estratégia Saúde da Família. Recursos humanos em saúde.

\begin{abstract}
\end{abstract}
Formation, provision and placement of doctors in the Family Health Strategy (ESF) are relevant points for the strengthening of Primary Care in Brazil. The objective is to evaluate the formation and factors related to the provision and placement of the doctors of ESF in a municipality from the Northeast Region of Brazil, establishing a comparison with dentists and nurses. This is a quantitative descriptive study with a questionnaire structured with 119 health professionals. The doctors presented

\footnotetext{
${ }^{1}$ Universidade Federal do Ceará (UFC). E-mail: brunoaguiarufc@hotmail.com.

2 Universidade Federal do Ceará (UFC).

${ }^{3}$ Universidade Federal do Ceará (UFC).
} 
good index (68\%) of specialization in family health, average time in the FHS of 5.2 years, besides considering the bonus of the public service examination the financial need the main reasons for participating in the FHS. $80 \%$ of the doctors belonged to the More Doctors Program (Programa Mais Médicos), which is the big difference between the non-medical professionals, who are predominantely public servants.

KEYWORDS: Primary health care. Health human resource training. Family Health Strategy. Health Workforce.

\section{INTRODUÇÃO}

O Sistema Único de Saúde (SUS) foi criado a partir das definições legais estabelecidas pela nova Constituição Federal do Brasil de $1988 .{ }^{1}$ Apesar de decorridas quase três décadas, a questão do provimento e da fixação dos profissionais médicos no Sistema Único de Saúde (SUS) permanece como um problema crônico a ser enfrentado. ${ }^{2}$

A não "submissão" da atuação dos profissionais médicos contribui drasticamente para o não alcance dos princípios do SUS: universalidade, equidade, atenção integral pelo reconhecimento dos determinantes sociais do processo saúdedoença e resolubilidade. Mesmo com a reorientação das políticas de saúde, como a Estratégia Saúde da Família (ESF) para a Atenção Básica, relegadas pelos sucessivos governos no período pós-constitucional, esse sistema consolida-se com baixa qualidade para atendimento da população pobre, que não pode pagar a saúde suplementar. ${ }^{3}$

Captação, formação e fixação de profissionais são condições necessárias para a consolidação da ESF como modalidade estruturante da Atenção Primária à Saúde no país. ${ }^{4} \mathrm{O}$ grande aumento do número de postos de trabalho, com a expansão de cobertura assistencial da Atenção Básica produzida pela progressiva implantação da ESF, torna esta temática importante não apenas na perspectiva de propor estratégias de provimento e de fixação dos profissionais de saúde, mas também aponta a necessidade de investir no processo de formação médica e de educação permanente dos trabalhadores do SUS de acordo com o modelo assistencial centrado no cuidado em saúde. ${ }^{5}$

Dentre os principais fatores que contribuem para a alta rotatividade dos médicos no SUS, em especial na Atenção Básica, podem ser listados: falta de condições materiais (medicamentos, insumos e infraestrutura das unidades de saúde); sobrecarga de 
trabalho; distância dos grandes centros urbanos; falta de capacitação para exercer a prática de generalista na ESF; e a presença de um mercado de trabalho dinâmico, sempre capaz de fornecer novas oportunidades. ${ }^{4,5}$ Embora o salário seja um dos atrativos para os médicos se apresentarem para contratação na ESF, este sozinho não tem garantido a fixação. Atribui-se ao fato de que, em geral, as melhores propostas remuneratórias são em áreas longínquas e pouco atrativas em termos de desenvolvimento econômico e sociocultural. ${ }^{6,7}$

Ressalte-se também que, a fim de aumentar os ganhos com seu trabalho, os médicos articulam vários empregos, exercendo a profissão em locais diferentes em um constante ir e vir, inclusive em variados municípios, contribuindo para a precarização da qualidade de suas vidas e, consequentemente, do sistema de saúde. ${ }^{2}$

Outra questão relacionada à maior dificuldade para compor e manter o profissional médico nas equipes de saúde da família diz respeito à incipiente regulação estatal sobre a profissão médica no Brasil, ao contrário do que ocorre em outros países. Austrália, França, Reino Unido e Suécia, por exemplo, adotam políticas de regulação do cadastro de médicos e suas especialidades através do controle da formação, por considerarem que o mercado de atenção à saúde não é um mercado livre e, desse modo, valorizam o papel da atenção primária nos seus sistemas de saúde. ${ }^{7}$

Outros tópicos relacionados à dificuldade de provimento e fixação de médicos também entram em debate. Faltam médicos no Brasil? É necessário importar médicos? Para Feurwerker, ${ }^{8}$ a resposta a ambas as perguntas é sim, dada a dramática escassez experimentada não somente na Atenção Básica e nos lugares distantes ou muito pobres, mas também na urgência/emergência, nos Centros de Atenção Psicossocial (CAPS), nos ambulatórios de especialidades e nas Unidades de Terapia Intensiva (UTIs). Porém, para se responder de quantos médicos precisamos e de que médicos precisamos, vai depender do modelo de atenção à saúde, dos arranjos tecnológicos de trabalho, do diálogo entre os trabalhadores de saúde e do tipo de formação (biologicista ou centrada no cuidado em saúde) que se pretende garantir aos futuros profissionais.

O Ministério da Saúde já implantou diversos programas na tentativa de consolidar o provimento e a fixação de profissionais médicos no SUS, muitos deles sofrendo forte influência política em períodos de sucessão presidencial. Em 1993, foi proposto o Programa de Interiorização do Sistema Único de Saúde (PISUS), defendendo 
a interiorização de uma equipe mínima além do médico; porém só durou 11 meses. Em 1994, a criação do Programa Saúde da Família (PSF) representou uma reorganização da Atenção Básica do SUS, mas com forte intenção também de melhorar a distribuição médica pelas diferentes regiões do país. Sete anos depois, o Programa de Interiorização do Trabalho em Saúde (PITS) representou uma estratégia de fortalecimento do PSF, ficando ativo até 2004, sendo encerrado com o início do governo Lula. ${ }^{9,10}$

Mais recentemente, dois programas ganharam destaque na área da saúde, buscando, dentre outros objetivos, o provimento e a fixação dos profissionais de saúde: Programa de Valorização do Profissional da Atenção Básica (Provab) e Programa Mais Médicos. O Provab foi criado pelo governo federal em 2011, com o objetivo de prover profissionais de saúde para as localidades com baixo Índice de Desenvolvimento Humano (IDH) e com população com pouca cobertura assistencial, destacando-se as seguintes ofertas do programa: pontuação de $10 \%$ nas provas de residência após avaliação do profissional e atividades estruturadas de educação a distância e supervisão. ${ }^{11}$

Já o Programa Mais Médicos foi introduzido no Brasil em 2013 como parte de uma série de medidas para combater as desigualdades de acesso à Atenção Básica resolutiva, incidindo sobre a falta de médicos em curto e médio prazo, ao associar o provimento com a ampliação e as mudanças na formação do profissional. ${ }^{12,13} \mathrm{O}$ programa foi estruturado em três eixos de ação. O primeiro é o investimento na melhoria da infraestrutura da rede de saúde, particularmente nas Unidades Básicas de Saúde (UBS). O segundo é a ampliação e as reformas educacionais dos cursos de graduação em Medicina e residência médica no país. Por último, a provisão emergencial de médicos, tanto brasileiros como estrangeiros, em áreas vulneráveis. ${ }^{3,12,14}$

Segundo o Cadastro Nacional de Estabelecimentos de Saúde (CNES), o Brasil contava no ano de 2016 com aproximadamente 15.164 equipes de Saúde da Família do Programa Mais Médicos. A região Nordeste apresenta 5.697 dessas equipes, sendo 1.283 no estado do Ceará. ${ }^{15} \mathrm{~A}$ concentração de profissionais médicos em determinadas regiões do país, a discrepância entre os percentuais de cobertura de Atenção Básica em determinadas regiões e a desproporção da quantidade de profissionais de alta e média complexidade com relação à quantidade de profissionais de Atenção Básica demonstram que o SUS não está cumprindo seu papel como ordenador e formador de 
recursos, conforme art. 200, inciso III, da Constituição Federal de $1988 .{ }^{16}$

Diante do exposto, o objetivo do presente trabalho é avaliar a formação e o provimento dos médicos da ESF em município da região metropolitana de um estado do Nordeste, estabelecendo um comparativo com dentistas e enfermeiros (profissionais não médicos).

\section{MATERIAIS E MÉTODOS}

Trata-se de estudo descritivo transversal sobre a formação e o provimento dos médicos da ESF realizado no período de dezembro de 2015 a janeiro de 2016 em um município localizado no estado do Ceará e pertencente à região metropolitana de Fortaleza.

Atualmente, o estado apresenta uma população estimada em aproximadamente 349.526 habitantes, em uma área de unidade territorial de $1.228 \mathrm{~km}^{2}$, segundo dados do último censo do Instituto Brasileiro de Geografia e Estatística (IBGE). ${ }^{17}$ A Atenção Básica é composta por 68 equipes da ESF funcionando em 44 UBS, dois CAPS e seis Núcleos de Apoio à Saúde da Família (NASF). ${ }^{15}$ Apresenta o Índice de Desenvolvimento Humano (IDH) no valor de 0,682. A proporção de médicos/mil habitantes e dentistas/mil habitantes corresponde respectivamente a 1,07 e $0,26{ }^{18}$

A população do estudo correspondeu ao quadro de todos os profissionais de saúde (médicos, dentistas e enfermeiros) integrantes da ESF do município. No total, foram 149 profissionais de saúde, divididos em 43 médicos, 46 dentistas e 60 enfermeiros, compondo 68 equipes selecionadas.

Os critérios de inclusão foram: ser profissional de saúde da equipe da ESF (médico, dentista ou enfermeiro); ter vínculo comprovável (servidor estatutário, celetista, contrato por tempo determinado ou participante de programas Provab/Mais Médicos) no CNES ou na Secretaria Municipal de Saúde da cidade. Os critérios de exclusão adotados foram a seleção do profissional para participação de teste piloto, que ocorreu previamente à pesquisa, através da escolha de duas equipes de maneira aleatória (sorteio), e o afastamento legal do exercício trabalhista (férias ou licença) no momento da coleta de dados.

O instrumento de coleta de dados consistiu na aplicação de questionário com 15 
perguntas com todos os profissionais de saúde da ESF do município. O questionário foi dividido em três partes: dados de identificação e aspectos sociodemográficos dos profissionais de saúde; características sobre a formação acadêmica e titulação; e informações da vida profissional/competências, habilidades e atribuições na ESF.

Para análise descritiva, as variáveis foram classificadas em quantitativas e qualitativas.

As definições das quantitativas foram:

- Idade: idade no momento do exame.

- Tempo decorrido da conclusão do curso de graduação: período (em anos) compreendido entre a conclusão do curso de graduação e a data do preenchimento do questionário.

- Tempo decorrido da conclusão do último curso de pós-graduação: período (em anos) compreendido entre a conclusão do último curso de pós-graduação e a data da realização da coleta.

- Tempo de trabalho na ESF: período compreendido entre o início de atuação na ESF e a data do preenchimento do questionário. Consideramos todas as experiências de trabalho na ESF, tanto em Caucaia como em municípios anteriores.

- Nota da graduação para a formação para a ESF: nota dos participantes a respeito de sua formação para o modelo da ESF.

As variáveis qualitativas utilizadas foram:

- Gênero: definição do participante como homem (masculino) ou mulher (feminino).

- Grau máximo de titulação: curso de maior titulação do participante, considerando a seguinte ordem crescente de hierarquia (graduação, residência, especialização, mestrado, doutorado, pós-doutorado).

- Cursos de pós-graduação: classificação dos cursos de pós-graduação dos participantes, enquadrando-os em uma das temáticas propostas pelo questionário.

- Motivo da escolha de curso de pós-graduação voltado para ESF: representação das principais causas responsáveis pela busca por parte dos participantes de 
cursos de pós-graduação voltados para a atuação na ESF.

- Razões para o trabalho na ESF: representação das principais causas para atuação dos participantes na ESF.

- Contribuição para a educação permanente dos profissionais de saúde: alternativas apresentadas pelos participantes para a melhoria da educação permanente na ESF.

Os dados coletados foram armazenados no banco de dados Epidata $3.1 \mathrm{e}$ analisados no pacote estatístico STATA 12.0. Para as variáveis qualitativas foram realizadas estatísticas descritivas simples (proporção). Para as variáveis quantitativas foram empregadas medidas de tendência central (média e mediana) e de dispersão (desvio padrão).

O projeto foi submetido e aprovado pelo Comitê de Ética e Pesquisa da Universidade Federal do Ceará (UFC), de acordo com a Resolução no 466/12 do Conselho Nacional de Saúde (CNS) com o parecer consubstanciado no 1.292.629/2015.

Aos participantes do estudo foram garantidos o cumprimento dos preceitos éticos da beneficência, da não maleficência, bem como o direito ao anonimato e sua autonomia quanto a participar da pesquisa sem qualquer prejuízo, podendo desistir a qualquer momento. Todos foram informados dos objetivos do estudo e da justificativa mediante apresentação do Termo de Consentimento Livre e Esclarecido (TCLE).

\section{RESULTADOS}

Dos 149 profissionais que participaram do estudo, 20,1\% (30) foram excluídos da pesquisa, sendo 22 recusas e oito profissionais que não participaram por estarem de férias ou licença no período da coleta de dados.

Dos 119 profissionais de saúde, 42\% (50) eram enfermeiros, 37\% (44) dentistas e 21\% (25) médicos. Verificou-se predominância do gênero feminino entre os profissionais de saúde, sendo 73,1\% (87) mulheres e 26,9\% (32) homens (Tabela 1). Em relação à idade, a média do estudo foi de 35 anos, variando entre 23 e 71 anos, ocorrendo pouca diferença quando se comparam as médias por profissão (Tabela 2).

A média do tempo decorrido do ano de conclusão da graduação foi de 9,7 anos, correspondendo ao ano de 2005. As médias do ano de término da graduação por 
profissão apresentaram valores próximos, sendo a categoria médica aquela que apresenta formação mais recente, seguida pelos enfermeiros e depois pelos dentistas (Tabela 2). Porém, a mediana demonstrou uma diferença considerável na dispersão desses dados entre as profissões, evidenciando que os médicos apresentaram uma tendência de serem recém-formados.

No estudo, verificou-se que $11,8 \%$ (14) dos profissionais concluíram o curso de graduação até 1994, sendo quatro médicos, sete dentistas e três enfermeiros (Tabela 2).

Dos 119 profissionais, 92,4\% (110) realizaram algum curso de pós-graduação, seja lato sensu ou stricto sensu, enquanto 7,6\% (9) ainda eram somente graduados (Tabela 1). A média do tempo decorrido do ano de conclusão deste curso foi de 4,6 anos, correspondendo ao ano de 2010 (Tabela 2). A mediana por categoria profissional possibilitou observar a dispersão do tempo decorrido da última pós-graduação, evidenciando que os médicos a realizaram mais recentemente, enquanto os enfermeiros foram os que concluíram a última pós-graduação há mais tempo.

A divisão dos profissionais por grau máximo de titulação (graduação, especialização, residência, mestrado acadêmico, mestrado profissional, doutorado e pós-doutorado) pode ser observada na Tabela 1. De modo geral, verificou-se predomínio de realização dos cursos de especialização, independentemente da categoria profissional, contabilizando 83,2\% (99) como especialistas. A residência ainda se mostra um curso pouco procurado entre os profissionais, mesmo com a criação da Residência Multiprofissional em Saúde da Família, tendo sido registrado baixo índice de residentes apenas entre médicos e enfermeiros.

Os cursos de pós-graduação stricto sensu apresentaram poucos representantes entre os profissionais de saúde, totalizando apenas 7,6\% (9) como mestres, doutores e/ou com pós-doutorado, composto por 11,3\% (5) dos dentistas e $8 \%$ (4) dos enfermeiros. Nenhum médico relatou ter concluído curso stricto sensu (Tabela 1).

Quanto à classificação dos cursos de pós-graduação realizados, percebeu-se que 50,4\% (60) dos profissionais têm especialização em Saúde da Família; enquanto 7,6\% (9) apresentam em Saúde Pública; e 4,2\% (5), em Saúde Coletiva. Quando se considera o número de participantes que apresenta pelo menos um desses três cursos, verificaramse $54,6 \%$ (65) dos profissionais, representando aqueles com formação voltada ao 
modelo da ESF. Observou-se uma maior busca por esses cursos principalmente por médicos (68\%) e enfermeiros (60\%), e menos pelos dentistas (40,9\%) (Gráfico 1).

Os cursos de pós-graduação voltados para especialidades clínicas também tiveram grande destaque, constatando-se que 49,6\% (59) dos profissionais apresentavam essa qualificação. Nesse caso, a classe odontológica, representada por 75\% (33) dos dentistas, colabora para a elevada estatística desse tipo de curso, sendo os demais representados por $40 \%$ (20) dos enfermeiros e $24 \%$ (6) dos médicos. Os cursos de Auditoria/Gestão em Saúde correspondem ao terceiro tipo mais apresentado, contabilizando $11,8 \%$ (14) dos profissionais. Em seguida, 10,1\% (12) dos participantes escolheram a opção "outros", para a qual foram citadas pós-graduações diversas, como: Medicina/Enfermagem/Odontologia do Trabalho, Ensino na Saúde, Ciências Básicas (microbiologia laboratorial) e Promoção da Saúde na Enfermagem (Tabela 1).

O tempo médio de trabalho na ESF foi de 6,2 anos, com variação de 1 (mínimo) a 15 (máximo) anos. Os médicos apresentaram uma média individual um pouco menor quando comparada ao tempo de dentistas e enfermeiros (Tabela 2). Esse tempo abrange todas as experiências de trabalho na ESF, tanto neste como em municípios anteriores.

Os profissionais também foram classificados quanto ao vínculo de trabalho estabelecido com a Prefeitura. "Concurso público" foi a forma que apresentou o maior percentual, contando com $74,8 \%$ (89) dos profissionais; seguida pela classificação "bolsista Mais Médicos", com 16,8\% (20) dos participantes. Os vínculos "contrato por tempo determinado" e "processo seletivo (celetista)" apresentaram respectivamente $5,9 \%$ (7) e 2,5\% (3) dos profissionais. O item "bolsista Provab" não foi assinalado no questionário (Tabela 1).

Em relação ao tipo de vínculo trabalhista separadamente por profissão, observou-se que a categoria médica apresentou $80 \%$ (20) dos profissionais como bolsistas do Programa Mais Médicos, enquanto apenas 16\% (4) eram concursados (Tabela 1). Em contraste, a Odontologia e a Enfermagem demonstram forte relação com o concurso público, observando-se $93,1 \%$ (41) dos dentistas e $88 \%$ (44) dos enfermeiros nessa classificação (Tabela 1 - pág. 106).

Outro questionamento tratado com os profissionais de saúde foi sobre as razões que os levavam a trabalhar na ESF. A “identificação com o modelo sanitário" e a 
"necessidade financeira" foram os principais motivos apresentados, tendo sido escolhidas respectivamente por $61,3 \%$ (73) e 40,3\% (48) dos participantes. A análise separada por categoria traz que os médicos consideram as duas principais razões a "bonificação para prova de títulos" e a "necessidade financeira", enquanto as opiniões de dentistas e enfermeiros coincidem com os dados gerais dos profissionais de saúde. As alternativas "falta de outra oportunidade no mercado de trabalho" e "estabilidade" foram pouco escolhidas pelos participantes (Tabela 2 - pág. 107).

A variável "nota atribuída à graduação para formação para a ESF" apresentou uma média de 7,8, trazendo uma variação de 3 (mínimo) a 10 (máximo). Não houve diferença significativa entre os valores das médias por profissão, porém a Enfermagem apresentou a melhor média (8,2); e a Odontologia, a pior $(7,1)$ (Tabela 2). A avaliação dos valores da mediana dessa variável por categoria permitiu verificar que médicos consideram sua graduação mais apropriada para a formação em ESF.

Dentre os fatores que contribuíram para a formação e a atuação na ESF, "disciplinas da graduação" e "estágios nos serviços de saúde", foram considerados os de maior impacto por 66,4\% (79) e 65,5\% (78) dos profissionais. "Manuais, cadernos e portarias da atenção básica" representou o terceiro fator mais escolhido, seguido por "cursos de pós-graduação" e "projetos de extensão, pesquisa ou monitoria". 
Tabela 1 -- Relação das variáveis qualitativas por categoria profissional: gênero, grau máximo de titulação, cursos de pós-graduação, vínculo, razões para trabalho na ESF, fatores que contribuíram para formação e atuação na ESF, sugestões para educação permanente profissional, em Caucaia (CE), 2016

\begin{tabular}{|c|c|c|c|c|}
\hline \multirow[t]{2}{*}{ Variáveis } & \multicolumn{3}{|c|}{ Categoria profissional } & \multirow[b]{2}{*}{$\begin{array}{c}\text { Total } \\
(n=119) \\
n(\%)\end{array}$} \\
\hline & $\begin{array}{l}\text { Médicos } \\
(\mathrm{n}=25) \\
\mathrm{n}(\%)\end{array}$ & $\begin{array}{l}\text { Dentistas } \\
(\mathrm{n}=44) \\
\mathrm{n}(\%)\end{array}$ & $\begin{array}{c}\text { Enfermeiros } \\
(\mathrm{n}=50) \\
\mathrm{n}(\%)\end{array}$ & \\
\hline \multicolumn{5}{|l|}{ Gênero } \\
\hline Masculino & $12(48,0)$ & $17(38,6)$ & $3(6,0)$ & $32(26,9)$ \\
\hline Feminino & $13(52,0)$ & $27(61,4)$ & $47(94,0)$ & $87(73,1)$ \\
\hline \multicolumn{5}{|l|}{ Titulação máxima } \\
\hline Graduação & $6(24,0)$ & $1(2,3)$ & $2(4,0)$ & $9(7,6)$ \\
\hline Especialização & $17(68,0)$ & $38(86,3)$ & $44(88,0)$ & $99(83,2)$ \\
\hline Residência & $2(8,0)$ & 0 & 0 & $2(1,7)$ \\
\hline Mestrado acadêmico & 0 & $2(4,5)$ & $3(6,0)$ & $5(4,2)$ \\
\hline Mestrado profissional & 0 & $2(4,5)$ & $1(2,0)$ & $3(2,5)$ \\
\hline Doutorado & 0 & 0 & 0 & 0 \\
\hline Pós-Doutorado & 0 & $1(2,3)$ & 0 & $1(0,8)$ \\
\hline \multicolumn{5}{|l|}{ Cursos de pós-graduação } \\
\hline Saúde da Família & $17(68,0)$ & $15(34,1)$ & $28(56,0)$ & $60(50,4)$ \\
\hline Saúde Pública & $1(4,0)$ & $3(6,8)$ & $5(10,0)$ & $9(7,6)$ \\
\hline Saúde Coletiva & 0 & $2(4,5)$ & $3(6,0)$ & $5(4,2)$ \\
\hline Especialidades Clínicas & $6(24,0)$ & $33(75,0)$ & $20(40,0)$ & $59(49,6)$ \\
\hline Auditoria/Gestão em Saúde & 0 & $9(20,4)$ & $5(10,0)$ & $14(11,8)$ \\
\hline Marketing em Saúde & 0 & 0 & 0 & 0 \\
\hline Economia ou MBA em Saúde & 0 & 0 & 0 & 0 \\
\hline Curso não relacionado à saúde & 0 & 0 & $1(2,0)$ & $1(0,8)$ \\
\hline Outros & $2(8,0)$ & $2(4,5)$ & $8(16,0)$ & $12(10,1)$ \\
\hline \multicolumn{5}{|l|}{ Vínculo } \\
\hline Concurso público (estatutário) & $4(16,0)$ & $41(93,1)$ & $44(88,0)$ & $89(74,8)$ \\
\hline Processo seletivo (celetista) & 0 & $3(6,8)$ & 0 & $3(2,5)$ \\
\hline Contrato de tempo determinado & $1(4,0)$ & 0 & $6(12,0)$ & $7(5,9)$ \\
\hline Bolsista Provab & 0 & 0 & 0 & 0 \\
\hline Bolsista Mais Médicos & $20(80,0)$ & 0 & 0 & $20(16,8)$ \\
\hline \multicolumn{5}{|l|}{ Razões para trabalho na ESF } \\
\hline $\begin{array}{l}\text { Identificação com o modelo } \\
\text { sanitário }\end{array}$ & $8(32,0)$ & $20(45,4)$ & $45(90,0)$ & $73(61,3)$ \\
\hline $\begin{array}{l}\text { Falta de outra oportunidade no } \\
\text { mercado de trabalho }\end{array}$ & 0 & $6(13,6)$ & $2(4,0)$ & $8(6,7)$ \\
\hline $\begin{array}{l}\text { Bonificação para prova de títulos } \\
\text { de residência ou concursos } \\
\text { públicos }\end{array}$ & $14(56,0)$ & $2(4,5)$ & $1(2,0)$ & $17(14,3)$ \\
\hline Necessidade financeira & $14(56,0)$ & $24(54,5)$ & $40(80,0)$ & $48(40,3)$ \\
\hline Estabilidade & 0 & $5(11,4)$ & $3(6,0)$ & $8(6,7)$ \\
\hline
\end{tabular}


(conclusão)

\begin{tabular}{|c|c|c|c|c|}
\hline \multirow[t]{2}{*}{ Variáveis } & \multicolumn{3}{|c|}{ Categoria profissional } & \multirow[b]{2}{*}{$\begin{array}{c}\text { Total } \\
(\mathrm{n}=119) \\
\mathrm{n}(\%)\end{array}$} \\
\hline & $\begin{array}{c}\text { Médicos } \\
(\mathrm{n}=25) \\
\mathrm{n}(\%)\end{array}$ & $\begin{array}{c}\text { Dentistas } \\
(n=44) \\
n(\%)\end{array}$ & $\begin{array}{c}\text { Enfermeiros } \\
(\mathrm{n}=50) \\
\mathrm{n}(\%)\end{array}$ & \\
\hline \multicolumn{5}{|l|}{$\begin{array}{l}\text { Fatores que contribuíram para } \\
\text { formação e atuação na ESF } \\
\text { Disciplinas da graduação }\end{array}$} \\
\hline Estágios nos serviços de saúde & $17(68,0)$ & $22(50,0)$ & $40(80,0)$ & $79(66,4)$ \\
\hline Projetos de extensão, pesquisa & $19(76,0)$ & $26(59,1)$ & $33(66,0)$ & $78(65,5)$ \\
\hline ou monitoria & $5(20,0)$ & $13(29,5)$ & $4(8,0)$ & $22(18,5)$ \\
\hline $\begin{array}{l}\text { Manuais, cadernos e portarias } \\
\text { da Atenção Básica } \\
\text { Cursos de pós-graduação }\end{array}$ & $12(48,0)$ & $13(29,5)$ & $29(58,0)$ & $54(45,4)$ \\
\hline \multirow[t]{2}{*}{ Outros } & $7(28,0)$ & $16(36,4)$ & $17(34,0)$ & $40(33,6)$ \\
\hline & $2(8,0)$ & $3(6,8)$ & $1(2,0)$ & $6(5,0)$ \\
\hline
\end{tabular}

Fonte: Elaborada pelos autores - 2017

Tabela 2 - Relação das variáveis quantitativas por categoria profissional: idade, tempo de conclusão da graduação, tempo de conclusão da pós-graduação, tempo de trabalho na ESF, nota atribuída à graduação para a ESF, em Caucaia (CE), 2016

\begin{tabular}{|c|c|c|c|c|c|c|c|c|c|c|c|c|}
\hline \multirow[t]{2}{*}{ Variável } & \multicolumn{3}{|c|}{$\begin{array}{l}\text { Médicos } \\
(n=25)\end{array}$} & \multicolumn{3}{|c|}{$\begin{array}{l}\text { Dentistas } \\
(n=44)\end{array}$} & \multicolumn{3}{|c|}{$\begin{array}{l}\text { Enfermeiros } \\
(n=50)\end{array}$} & \multicolumn{3}{|c|}{$\begin{array}{c}\text { Total } \\
(n=119)\end{array}$} \\
\hline & $\begin{array}{l}M \\
\text { (D) }\end{array}$ & Md & $\begin{array}{l}\mathrm{Mn} \\
\mathrm{Mx}\end{array}$ & $\begin{array}{l}M \\
\text { (D) }\end{array}$ & Md & $\begin{array}{l}\mathrm{Mn} \\
\mathrm{Mx}\end{array}$ & $\begin{array}{l}M \\
\text { (D) }\end{array}$ & Md & $\begin{array}{l}\text { Mn } \\
M x\end{array}$ & $\begin{array}{l}M \\
\text { (D) }\end{array}$ & Md & $\begin{array}{l}\mathrm{Mn} \\
\mathrm{Mx}\end{array}$ \\
\hline \multirow[t]{2}{*}{ Idade } & 34,4 & 30 & 25 & 35,0 & 33 & 28 & 35,2 & 33 & 23 & 35,0 & 32 & 23 \\
\hline & $\pm 11,3$ & & 71 & $\pm 7,0$ & & 55 & $\pm 8,1$ & & 65 & $\pm 8,5$ & & 71 \\
\hline
\end{tabular}

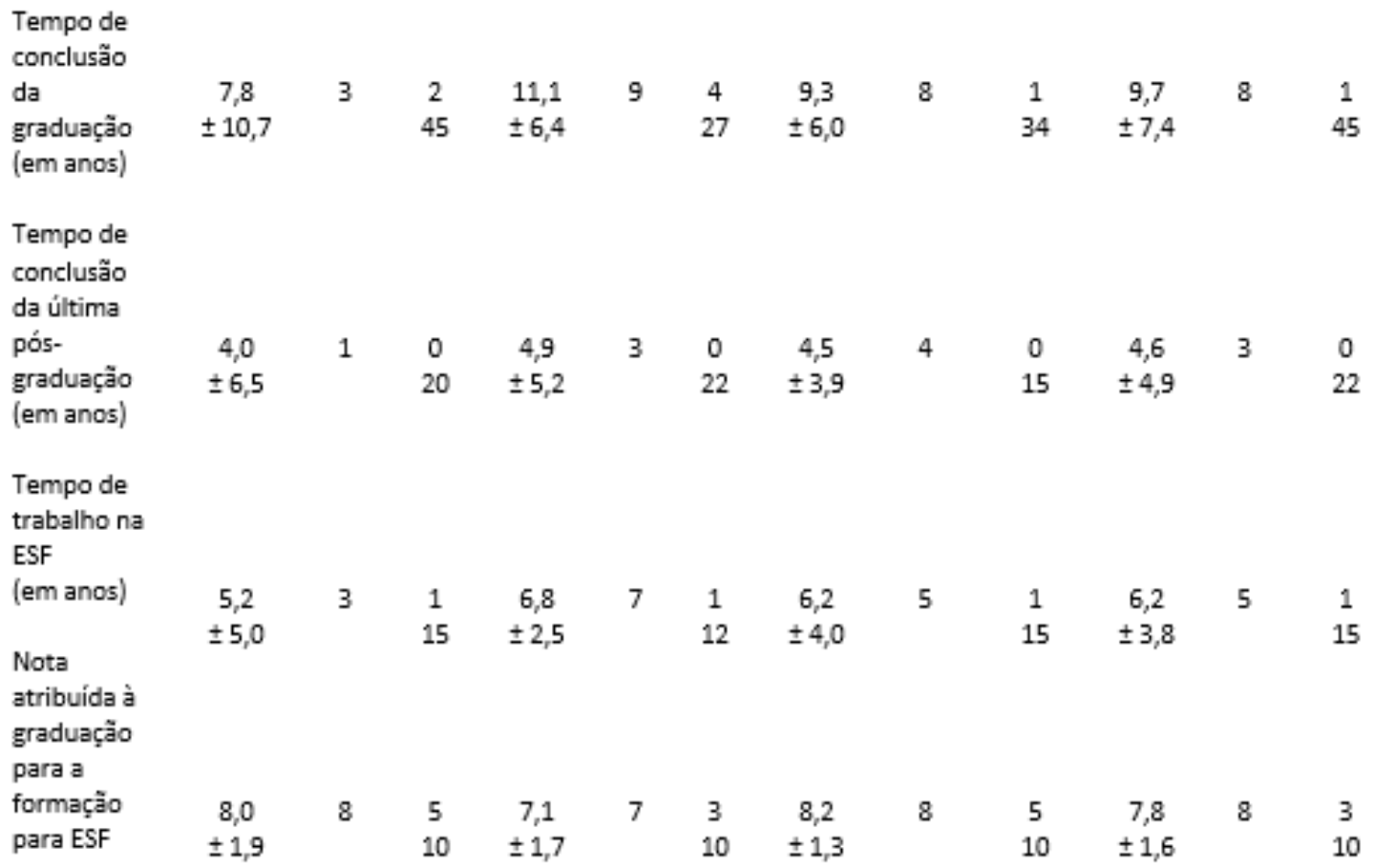

Legenda: $\mathrm{M}$ = média; $\mathrm{D}$ = desvio padrão; $\mathrm{Md}$ = mediana; $\mathrm{Mn}=$ valor mínimo; $\mathrm{Mx}=$ valor máximo

Fonte: Elaborada pelos autores - 2017 
Gráfico 1 - Motivos da realização dos cursos de Saúde da Família, Saúde Pública e/ou Saúde Coletiva pelos profissionais de saúde, em Caucaia (CE), 2016

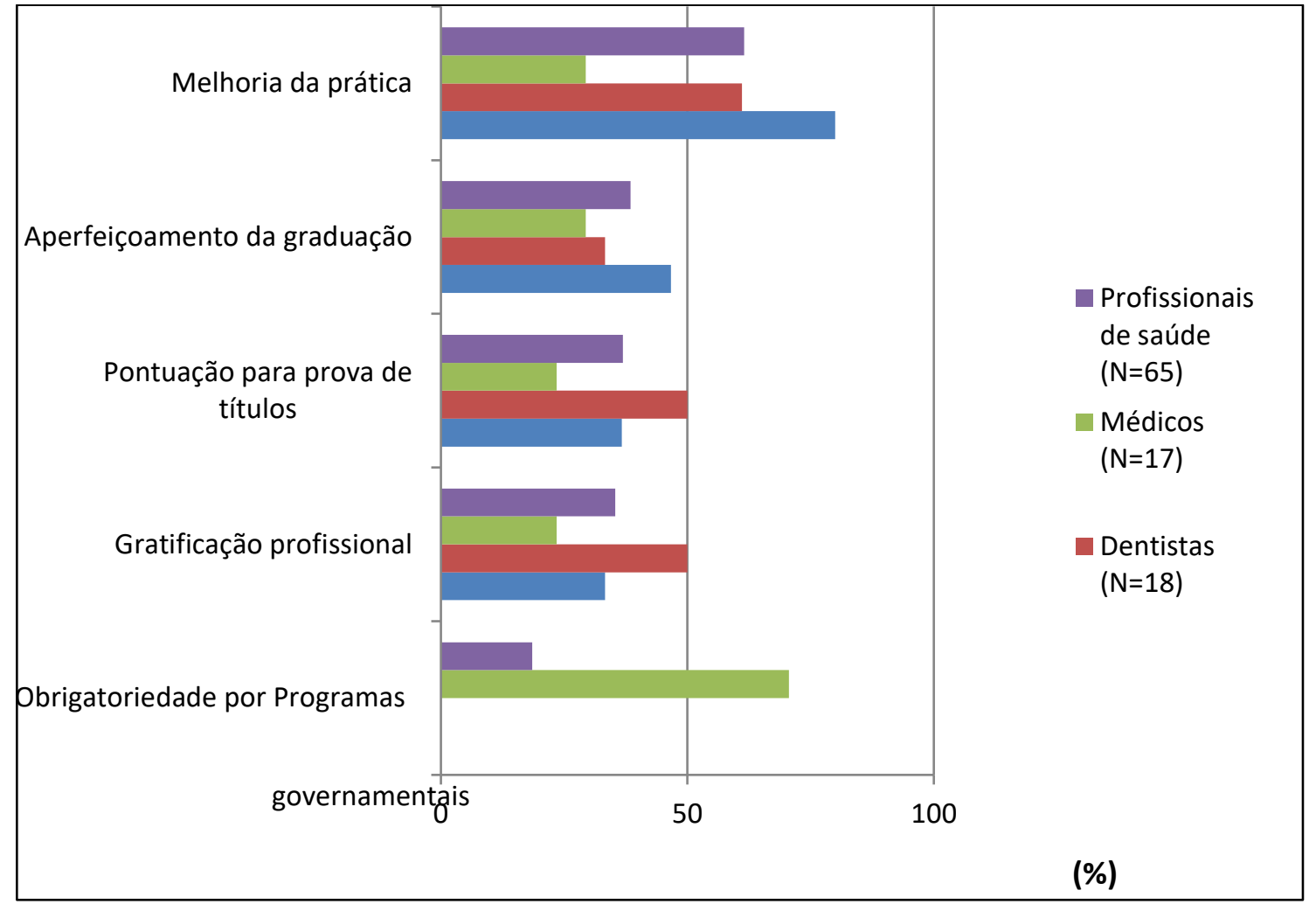

Fonte: Elaborada pelos autores - 2017

\section{DISCUSSÃO}

A formação, o provimento e a fixação dos profissionais de saúde tanto na ESF como nos serviços de saúde do SUS é tema contínuo de investigação de estudos na área de saúde pública. Esse interesse se deve à necessidade de compreensão do processo que vai desde a formação profissional até sua integração nos serviços de saúde, o que é de extrema relevância para a consolidação de qualquer modelo de atenção à saúde.

O trabalho encontrou como principais achados uma série de características para os profissionais de saúde da ESF com a seguinte predominância: gênero feminino, adultos jovens, concursados, bom tempo de trabalho na ESF, formação da graduação recente, interesse pela educação continuada, especialistas (principalmente em Saúde da Família e cursos voltados para especialidades clínicas) e profissionais identificados com o modelo sanitário.

Observou-se uma menor representatividade da categoria médica quando comparada às outras classes, tendo como justificativa o fato do desligamento ao longo 
do ano de grande parte desses profissionais com o município devido ao abandono do Programa Mais Médicos, corroborando com outros estudos $5,6,16,19,20$ que também descreveram alta rotatividade dos médicos na ESF.

Considerando que a criação do PSF ocorreu em 1994, pode-se afirmar que profissionais graduados antes disso não tiveram uma formação direcionada para a atuação nesse modelo assistencial em razão de sua inexistência, devendo a aquisição dos conhecimentos necessários para o exercício atual na ESF ter ocorrido através da educação continuada por meio de capacitação e/ou atualização em cursos de pósgraduação. $^{21}$

É necessário investir principalmente na qualificação do acesso e na formação do profissional da ESF voltada para o modelo sanitário, incluindo o reconhecimento dos especialistas na área de atenção primária à saúde e a instituição de um programa de educação continuada que alcance todos os profissionais. ${ }^{22} \mathrm{~A}$ educação permanente é fundamental para a aproximação do profissional com o SUS e a ESF, ${ }^{21}$ o que foi constatado na pesquisa através da preocupação por parte das três profissões da saúde em aderir ao processo de educação permanente e continuada após o curso de graduação.

A contribuição da graduação para a formação para ESF vem sendo fortemente influenciada pelas Diretrizes Curriculares Nacionais dos cursos de Medicina, Odontologia e Enfermagem. ${ }^{24,25}$ No caso do curso de Medicina, atualizações recentes foram instituídas pela Resolução CNE/CES no 3/2014. ${ }^{26}$ As diretrizes evidenciam que o profissional de saúde graduado tenha uma formação generalista, humanística, crítica e reflexiva para atuar em todos os níveis de atenção em saúde. Estimula também a compreensão da realidade social, cultural e econômica da população de seu meio, direcionando a atuação para a transformação da realidade em benefício da sociedade.

Em relação ao tipo de vínculo trabalhista, a realização de concurso público e o processo seletivo para captar recursos humanos à ESF demonstra a decisão e a vontade político-administrativa de consolidação da Atenção Básica, representando formas de se evitar a precarização do trabalho na ESF. ${ }^{4}$

Magnago ${ }^{19}$ relata em seu estudo que a contratação de profissionais de modo indireto gera conflitos entre servidores públicos municipais já atuantes na ESF, principalmente quando os contratados recebem salários superiores aos estatutários. 
Acrescenta que muitos gestores enxergam a contratação indireta de recursos humanos como alternativa de driblar o "comodismo" dos funcionários públicos, em razão de a estabilidade do emprego ser associada à ineficácia e a baixos índices de produtividade. Processos seletivos simplificados com remuneração acima do mercado, garantia dos direitos trabalhistas, abonos salariais e flexibilização de carga horária podem ser estratégias importantes para atração e fixação profissional, apesar da desvantagem da não estabilidade.

Importante ressaltar que, quando se analisaram os vínculos trabalhistas separadamente por profissão em nosso estudo, houve grandes discrepâncias. O fato de a grande parte da categoria médica ser do Programa Mais Médicos pode colaborar para a rotatividade de médicos na ESF e para a fragilização da relação equipe-comunidade em virtude da precarização de direitos trabalhistas e relações de vínculo com curto tempo de vigência preestabelecido. Em contraste, o alto índice de concursados nas áreas de Odontologia e de Enfermagem demonstra forte contribuição para a consolidação do profissional no território da ESF e a longitudinalidade da relação equipe-comunidade, conforme preconizado pela Política Nacional de Atenção Básica. ${ }^{13,20,26}$

Ademais, o grande percentual de médicos com especialização em Saúde da Família pode ter como justificativa o enquadramento desses profissionais como "bolsistas Mais Médicos". A partir de 2013, o programa trouxe a obrigatoriedade desse curso ou de especialização em Atenção Primária à Saúde em parceria com instituições públicas de Ensino Superior como eixo de formação para as políticas de saúde do SUS. ${ }^{12,14}$

A "identificação com o modelo sanitário" e a "necessidade financeira" foram relatadas nos questionários como as principais razões para o trabalho na ESF, retratando a afinidade profissional com o modelo da ESF aliada à garantia de seu sustento remuneratório. Fazendo uso de dito popular, significa que os profissionais de saúde estão "ganhando dinheiro (retorno financeiro) e fazendo o que gostam (retorno pessoal e profissional)". O estudo de Ney e Rodrigues ${ }^{7}$ cita como as principais razões para a permanência dos médicos na ESF a identificação com a filosofia da estratégia, a vocação profissional e a possibilidade de ajudar a comunidade. 


\section{CONSIDERAÇÕES FINAIS}

A formação dos profissionais de saúde vem se tornando mais adequada para a atuação na ESF. Esse processo tem relação com as diretrizes curriculares nacionais e a oferta de cursos de pós-graduação voltados para a prática nesse modelo da atenção básica, por vezes obrigatórios, como no caso de bolsistas do Programa Mais Médicos.

Conclui-se que, quanto ao provimento e à fixação dos profissionais de saúde, dentistas e enfermeiros apresentaram postura mais consolidada na ESF diante de médicos, constatada inclusive pelo vínculo de estabilidade. Os médicos ainda demonstraram ver na atenção básica uma situação transitória de trabalho, representando uma oportunidade de bonificação para prova de residência e uma garantia de remuneração.

\section{REFERÊNCIAS}

1. Brasil. Lei no 8.080, de 19 de setembro de 1990. Dispõe sobre as condições para a promoção, proteção e recuperação da saúde, a organização e o funcionamento dos serviços correspondentes e dá outras providências. Diário Oficial da União, 19 set 1990.

2. Maciel RH, Santos JBF, Sales TB, Alves MAA, Luna AP, Feitosa LB. Multiplicidade de vínculos de médicos no Estado do Ceará. Rev Saúde Pública. 2010;44(5), 950-6.

3. Costa AM, Girardi SN, Rocha VXM, Almeida ER, Santos LMP. Mais (e melhores) médicos Tempus Actas de Saúde Colet. 2015; 9(4), 175-181.

4. Lopes EZ, Bousquat AEM. Fixação de enfermeiras e médicos na Estratégia de Saúde da Família, município de Praia Grande, São Paulo, Brasil. Rev Bras Med Fam Comunidade. 2011; 6(19): 118-24.

5. Carvalho MS, Sousa MF. Como o Brasil tem enfrentado o tema provimento de médicos? Interface. 2013;17(47): 913-26.

6. Campos CVA, Malik AM. Satisfação no trabalho e rotatividade dos médicos do Programa de Saúde da Família. Rev. Adm. Pública. 2008; 42:347-68.

7. Ney MS, Rodrigues PHA. Fatores críticos para fixação do médico na Estratégia de Saúde da Família. Physis Revista de Saúde Coletiva. 2012; 22 (4): 1293-1311.

8. Feurwerker LCM. Médicos para o SUS: gestão do trabalho e da educação na saúde no olho do furacão! Interface. 2013; 17(47):929-30.

9. Filho R, Branco MAF. Rumo ao interior: médicos, saúde da família e mercado de trabalho. Cad. Saúde Pública. 2009;11(24):2531-2535. 
10. Maciel Filho R. Estratégias para distribuição e fixação de médicos em sistemas nacionais de saúde [Tese Doutorado em Política, Planejamento e Administração em Saúde]. Rio de Janeiro (RJ): Instituto de Medicina Social e Preventiva, Universidade do Estado do Rio de Janeiro, 2007.

11. Ministério da Saúde (Brasil). Portaria Interministerial MEC/MS no 2.087 de 01 de setembro de 2011. Institui o Programa de Valorização da Atenção Básica - PROVAB. Diário Oficial da União: 02 set 2011.

12. Brasil. Lei no 12.871 de 22 de outubro de 2013. Institui o Programa Mais Médicos. Diário Oficial da União: 23 out 2013.

13. Santos LMP, Costa AM, Girardi SN. Programa Mais Médicos: uma ação efetiva para reduzir iniquidades em saúde. Ciência \& Saúde Coletiva. 2015; 20(11):3547-3552.

14. Oliveira FP, Vanni T, Pinto HA, Santos JTR, Figueiredo AM, Araújo SQ, Matos MFM, Cyrino EG. Mais Médicos: um programa brasileiro em uma perspectiva internacional. Interface. 2015; 19(54): 623-34.

15. Cadastro Nacional dos Estabelecimentos de Saúde. Consulta de equipes. [Acesso em 29 mar 2016]. Disponível em: http://cnes2.datasus.gov.br/Lista_Tot_Equipes.asp.

16. Cardoso JR. Os desafios da Atenção Básica para consolidação do SUS e da garantia dos direitos sociais à saúde. Cad. IberAmer. Direito. Sanit. 2013; 2 (2), 543-558.

17. Instituto Brasileiro de Geografia e Estatística. População. Censo 2010. [Acesso em 29 mar 2016]. Disponível em: http://censo2010.ibge.gov.br/.

18 . Instituto de Pesquisa e Estratégia Econômica do Ceará. IPECE, 2016. Disponível em: <http://www.ipece.ce.gov.br/publicacoes/perfil_basico/pbm2015/Caucaia.pdf>. Acesso em 02 de agosto de 2016.

19. Magnago C. Gestão do trabalho na Estratégia Saúde da Família em municípios do Estado do Rio de Janeiro com mais de 500 mil habitantes: o caso do Rio de Janeiro e Duque de Caxias. 2012. 175f. Dissertação (Mestrado em Saúde Coletiva) - Instituto de Medicina Social, Universidade do Estado do Rio de Janeiro, Rio de Janeiro, 2012.

20. Giovani MSP, Vieira CM. Longitudinalidade do cuidado diante da rotatividade de profissionais na Estratégia Saúde da Família. RECIIS - R. Eletr. de Com. Inf. Inov. Saúde. 2013; 7(4), 1-14.

21. Villalba JP, Madureira PR, Barros NF. Perfil profissional do cirurgião-dentista para atuação no Sistema Único de Saúde (SUS). Rev Inst Ciênc Saúde. 2009; 27(3): 26268.

22. Castro RCLC, Knauth DRK, Harzeim E, Hauser L, Duncan BB. Avaliação da qualidade da atenção primária pelos profissionais de saúde: comparação entre diferentes tipos de serviços. Cad. Saúde Pública. 2012; 28(9),1772-84.

23. Brasil. Resolução CNE/CES no 3, de 7 de novembro de 2001. Diretrizes Curriculares Nacionais do Curso de Graduação em Enfermagem. Diário Oficial da União, 7 nov 2001.

24. Brasil. Resolução CNE/CSE no 3, de 19 de fevereiro de 2002. Diretrizes Curriculares Nacionais do Curso de Graduação em Odontologia. Diário Oficial da União, 19 fev 
2002.

25. Brasil. Resolução CNE/CES no 3, de 20 de junho de 2014. Diretrizes Curriculares Nacionais do Curso de Graduação em Medicina. Diário Oficial da União, 20 jun 2014.

26. Ministério da Saúde (Brasil). Secretaria de Atenção à Saúde. Departamento de Atenção Básica. Política Nacional de Atenção Básica. Série E, Legislação em saúde. Brasília (DF): MS, 2012.

Submissão: dezembro de 2017.

Aprovação: maio de 2018. 